\title{
Charged Tangles of Quantized Vortices in Superfluid ${ }^{4} \mathrm{He}$
}

\author{
A.I. Golov • P.M. Walmsley • P.A. Tompsett
}

Received: 15 September 2010 / Accepted: 30 September 2010 / Published online: 20 October 2010

(C) Springer Science+Business Media, LLC 2010

\begin{abstract}
We show that turbulence in superfluid ${ }^{4} \mathrm{He}$ at low temperatures can be generated and probed by injected ions trapped on vortex cores. The results of our recent experiments, in which negative ions were injected during short and long periods, in different quantities, and into different applied electric fields, are outlined. Three very different mechanisms of vortex-assisted transport of trapped ions were observed: one is on isolated vortex rings while two others are associated with tangles of vortex lines. It seems there are two different types of vortex tangles that can be characterized by the velocity of ion motion through them: a drifting polarized tangle in a low-dissipation state that mainly advects trapped ions, and a more isotropic tangle in a highly-dissipative state, sustained by a continuous forcing by the ion current in an applied electric field, through which trapped ions move rapidly.
\end{abstract}

Keywords Superfluidity · Superfluid helium · Quantized vortex · Quantum turbulence · Vortex tangle $\cdot$ Low temperature - Injected ion · Trapped ion · Charged vortex

\section{Quantum Turbulence at $T<1 \mathrm{~K}$ and the Need of New Methods of Forcing and Probing}

The dynamics of quantized vortices in superfluids are of great interest, especially when there are many such lines interacting [1-3]. When dissipative forces are small, and fluctuations of the superfluid flow generated by a tangle of quantized vortices involve a broad range of length scales, we call this Quantum Turbulence (QT). Historically, QT was discovered in superfluid ${ }^{4} \mathrm{He}$ at temperatures above $1 \mathrm{~K}$ where the 
drag on vortex lines caused by the relative motion of the normal and superfluid component (counterflow) can be used to force and detect QT [4-8]. The convenience of the counterflow as a means of generating QT is hard to underestimate: a heater-driven "fountain pump" does not require moving parts and is also capable of generating such an important and exotic type of QT as Vinen (i.e. "unstructured") turbulence $[9,10]-$ in which the dominant turbulent flow is on the quantum length scales $\leq \ell$ (where $\ell$ is the mean distance between vortices). An alternative to this regime is "quasi-classical" turbulence in which energy-dominating eddies are present at large length scales encompassing many quantized vortex lines; to describe flow dynamics on these scales, $\gg \ell$, one can neglect the discreteness of vorticity and hence recover the essentially Navier-Stokes fluid dynamics at high Reynolds numbers albeit with a somehow modified dissipative term $[9,11]$.

Of special interest is the limit of low temperatures $\left(T<0.4 \mathrm{~K}\right.$ for liquid $\left.{ }^{4} \mathrm{He}\right)$ $[12,13]$ where the normal component becomes dilute and no longer exerts an observable drag (and hence, damping) on vortex lines. As a result, the only available degrees of freedom of the superflow, the spatial configuration of vortex lines in the tangle, are free to evolve. This evolution mostly follows the Helmholtz laws of the ideal incompressible vortex dynamics: segments of vortex lines flow with the local superfluid velocity generated by all vortex lines (the leading contribution often being the self-induced velocity from the curved segment itself, especially for segments with high curvatures that are abundant in the presence of frequent vortex reconnections, and especially when the damping due to mutual friction vanishes) [14, 15]. Deviations from the inviscid incompressible hydrodynamics only occur when distances comparable to the vortex core radius, $a_{0} \sim 1 \AA$, come into play: either when vortex lines approach each other and reconnect or when high-frequency waves on vortex lines convert the flow energy into sound. One hence confronts an enormous range of length scales, typically between centimeters and nanometers, in which the dynamics of QT is approximately conservative. The rate of redistribution of energy from larger length scales to smaller and finally very small dissipative scales $[11,16]$ is controlled by the interactions between vortices, including their reconnections. Another important benefit of low temperatures is that the normal component no longer complicates the picture by its own turbulent dynamics-which is often the case in ${ }^{4} \mathrm{He}$ at $T>1 \mathrm{~K}$. This is why QT in the $T=0$ regime is often referred to as "pure quantum turbulence". However, there is also an experimental challenge: in the absence of the normal component, counterflow can no longer be employed for generating and detecting QT.

Very soon after the first indirect observations of the existence of quantized vortex lines through their interaction with counterflow [4-8, 17], injected ions were found to be extremely useful tools for detecting $[18,19]$ and forcing $[20]$ vortex lines. This eventually lead to the spectacular confirmation of the ideal dynamics of isolated quantized vortex rings [20] and direct visualization of rectilinear vortex lines in a rotating container [21]. There were observations that ion injection can result in generation of vortex tangles [22], and measurements of ion current can be used for detection of QT [23].

An excess electron, injected into the strongly-repelling liquid helium environment, quickly self-localizes in a cavity of radius $\sim 19 \AA$, from which all helium atoms are expelled [24-26]. We call such a bubble containing an electron a "negative ion". Under Bernoulli force such an object is then strongly attracted to the core of the vortex 
line. The depth of the trapping potential well is quoted [15] as $\sim 100 \mathrm{~K}$ (a theoretical value based on a model of a straight rigid vortex line) and $\sim 50 \mathrm{~K}$ (an experiment interpreted using a simplified model); hence, the lifetime of trapped negative ions is substantial already at $T<1.7 \mathrm{~K}$. It is expected that, at temperatures down to some $0.8 \mathrm{~K}$, the ions are relatively easy to capture by vortices [27] and virtually impossible to detach from them provided the electric field is not excessively high. There are still plenty of unknowns about the details of the ion-vortex interaction and behavior (see the discussion by Pi et al. [26] and references therein), especially with low damping at low temperatures and when the ion and vortex are subject to external force and perturbations. For instance, the experimental mobility of ions along vortex lines was found to greatly exceed a theoretical calculations of the effect of scattering of thermal Kelvin waves by an ion in the vicinity of the vortex core [28, 29]. It is also unknown whether the capture of bare ions by vortex lines can be efficient at low temperatures [27]. However, we do know that, at pressures below 10 bar (i.e. when its radius is the largest), a bare negative ion does not stay alone long. Even in tiny electric fields it quickly accelerates to the velocity at which a vortex ring nucleates within a small fraction of the ion drift time through an experimental cell [30], and then gets trapped by the vortex ring it just created. This is why in the experiments described below the pressure was kept as low as 0.1 bar-so that each ion is always trapped by either a vortex ring or an entangled vortex loop. Some insight into the particle-vortex dynamics can be gained from the theory for micron-sized spheres interacting with quantized vortex lines (see [31,32] and references therein)_-developed to interpret recent experiments for visualization of vortex lines in superfluid ${ }^{4} \mathrm{He}$ [33].

In Manchester, negative ions were recently used for the investigations of QT of different spectra in ${ }^{4} \mathrm{He}$ in the $T=0$ limit $[10,13,34,35]$. To measure the length of vortex lines per volume $L$, singly-charged vortex rings of a selected radius $R$ between 1 and $10 \mu \mathrm{m}$ were sent through the tangle, and measurements of their losses - due to the interactions with the tangle characterized by the geometrical scattering diameter $\sim R$ $[36,37]$ - were used to determine $L$. Also, vortex tangles of apparently Vinen type were generated by short injections of ions [10]. The latter demonstrates that trapped ions can be efficiently used to drive turbulence at $T<1 \mathrm{~K}$, where counterflow cannot be used.

The aim of this paper is to attract interest of both theoreticians and experimentalists to the subject and techniques of charged QT. We briefly outline our on-going investigations of the dynamics of charged vortices and their tangles, provide readers with important background information on injected ions and vortices, and discuss some speculative ideas how to interpret the observed effects. Detailed accounts of the experiments will be published elsewhere.

\section{Injected Ions and Quantized Vortices}

\subsection{Charged Tangles}

Let us begin from constructing a simple continuous model of the dynamics of a homogeneous charged tangle. The tangle is characterized by a uniform density $L=\ell^{-2}$ and 
drift velocity $\mathbf{v}_{L}$, that for random tangles (when the large-scale flow of the superfluid component is absent) is related to the curvature-weighted polarization of binormals to vortex segments $\mathbf{I}_{l}$ [14] as $\mathbf{v}_{L}=(4 \pi)^{-1 / 2} \Lambda \kappa L^{1 / 2} \mathbf{I}_{l}$. The trapped charge, assumed to be uniformly distributed along the vortex line, has volume density $q$ and drifts with velocity $\mathbf{v}_{q}$. The electric current density is thus $\mathbf{j}=q \mathbf{v}_{q}$. The externally-applied electric field $\mathbf{E}$ is constant in time.

We should make several reservations. Firstly, we will assume that the electric field self-induced by the trapped charge, $\sim q \sigma \epsilon_{0}^{-1}$, is small compared to the applied field $E$ :

$$
q \sigma \ll \epsilon_{0} E,
$$

where $\sigma$ is the spatial spread of the charged tangle, and we took the dielectric permeability of liquid helium $\epsilon \approx 1$. For $E=10 \mathrm{~V} \mathrm{~cm}^{-1}$ and $\sigma=1 \mathrm{~cm}$, this makes $q \ll 1 \mathrm{pC} \mathrm{cm}^{-3}$. Secondly, for the case of high $q$ and low $L$, such that the ion-ion interaction is mainly one-dimensional (when the average distance between neighboring ions $\delta \sim e L q^{-1}$ becomes much smaller than the size of typical vortex segments, $\delta \ll \ell$ i.e. $q \gg e L^{3 / 2}$, which is usually the case), $\delta$ should not be too small, otherwise the energy of the Coulomb repulsion per unit length, $\sim e^{2} \epsilon_{0}^{-1} \delta^{-2}$, will start affecting the line tension, $\sim \rho_{\mathrm{s}} \kappa^{2} \Lambda$ (where $\Lambda=\ln \ell a_{0}^{-1} \approx 15 \approx$ const). In other words, the critical ion-ion separation on a line is $\delta_{0} \sim e \kappa^{-1}\left(\epsilon_{0} \rho \Lambda\right)^{-1 / 2} \approx 120 \AA$, and

$$
q \ll\left(\epsilon_{0} \rho_{\mathrm{s}} \Lambda\right)^{\frac{1}{2}} \kappa L=\frac{e}{\delta_{0}} L .
$$

For $L=10^{4} \mathrm{~cm}^{-2}$, this means $q \ll 1 \mathrm{nC} \mathrm{cm}^{-3}$. We see that a tangle possessing a trapped charge of density $q$ cannot decay below certain minimal density

$$
L_{\min } \sim \frac{q}{\kappa\left(\epsilon_{0} \rho_{\mathrm{S}} \Lambda\right)^{\frac{1}{2}}}=\frac{\delta_{0}}{e} q .
$$

For our typical experimental vortex densities $L \sim 10^{2}-10^{4} \mathrm{~cm}^{-2}$, charge densities $q \sim 10^{-13}-10^{-11} \mathrm{C} \mathrm{cm}^{-3}$, electric fields $E \sim 10-100 \mathrm{~V} \mathrm{~cm}^{-1}$ and spatial spread of $\sigma \sim 1 \mathrm{~cm}$, these constrains are usually satisfied.

\subsection{Dynamics of Charged Tangles}

To get some insight, let us turn to the counterflow-driven QT at sufficiently low temperatures so that the mutual friction is weak enough not to slow down the vortex tangle. In a developed steady state with homogeneous velocities of normal and superfluid components $\mathbf{v}_{\mathrm{n}}$ and $\mathbf{v}_{\mathrm{s}}$ (state $T$ II in Tough's classification [38]), the rate of work by the mutual friction force $\mathbf{f}_{\mathrm{mf}}$ per unit volume is

$$
\dot{\mathcal{E}_{+}}=\mathbf{f}_{\mathrm{mf}} \cdot \mathbf{v}_{L}=\left(\rho_{\mathrm{s}} \kappa \alpha L \mathbf{v}_{\mathrm{ns}}\right) \cdot\left(\frac{\Lambda}{4 \pi} \kappa L^{1 / 2} \mathbf{I}_{l}\right)
$$

where $\mathbf{v}_{\mathrm{ns}}=\mathbf{v}_{\mathrm{n}}-\mathbf{v}_{\mathrm{s}}$ and $\alpha(T)$ is the dimensionless mutual friction parameter. The rate of energy dissipation is [2]

$$
\dot{\mathcal{E}_{-}}=-\rho_{\mathrm{s}} v^{\prime}(\kappa L)^{2}
$$


where the "effective kinematic viscosity" $v^{\prime}(T)$ might depend on the type of flow; for instance, at $T \rightarrow 0, v^{\prime} \approx 0.1 \kappa$ for Vinen turbulence [10] but can be much smaller for polarized tangles [34].

For unstructured tangles, the total energy is stored in the quantized flow round individual vortices:

$$
\mathcal{E}=\frac{\Lambda}{4 \pi} \rho_{\mathrm{s}} \kappa^{2} L
$$

The energy balance, $\dot{\mathcal{E}}=\dot{\mathcal{E}_{+}}+\dot{\mathcal{E}_{-}}$, results in the Vinen equation $[4-8,14]$

$$
\dot{L}=\alpha L^{3 / 2} \mathbf{I}_{l} \cdot \mathbf{v}_{\mathrm{ns}}-\frac{4 \pi}{\Lambda} v^{\prime} L^{2} .
$$

Its solution for steady-state $\dot{L}=0$,

$$
L=\left(\frac{\Lambda \alpha}{4 \pi v^{\prime}} \mathbf{I}_{l} \cdot \mathbf{v}_{\mathrm{ns}}\right)^{2},
$$

was found to describe both experimental and theoretical observations of steady-state vortex tangles [4-8, 14, 38, 39].

It seems plausible that the volume electrostatic force $\mathbf{f}_{q}=q \mathbf{E}$ on the trapped charge of density $q$ in field $\mathbf{E}$ can play a similar role to that of $\mathbf{f}_{\mathrm{mf}}$ in the counterflow field $\mathbf{v}_{\mathrm{ns}}$. This is especially important at low temperatures, $T<1 \mathrm{~K}$, when counterflow forcing becomes ineffective. We can compose an analog of the Vinen equation suitable for the case of forcing the tangle by moving the trapped charge at a current density $\mathbf{j}=q \mathbf{v}_{q}$ in an applied field $\mathbf{E}$. The energy production term $\dot{\mathcal{E}_{+}}$becomes:

$$
\dot{\mathcal{E}_{+}}=(q \mathbf{E}) \cdot \mathbf{v}_{q}=\mathbf{j} \cdot \mathbf{E} .
$$

We thus arrive at

$$
\frac{\Lambda}{4 \pi} \dot{L}=\frac{\mathbf{j} \cdot \mathbf{E}}{\rho_{\mathrm{s}} \kappa^{2}}-v^{\prime} L^{2} .
$$

For instance, for a steady-state, $\dot{L}=0$, it predicts

$$
\kappa L=\left(\frac{\mathbf{j} \cdot \mathbf{E}}{\rho_{\mathrm{s}} \nu^{\prime}}\right)^{1 / 2} .
$$

For our typical experimental values of $I \sim 10^{-12}$ A, $\sigma \sim 1 \mathrm{~cm}$ (i.e. $j \sim I \sigma^{-2} \sim$ $10^{-12} \mathrm{~A} \mathrm{~cm}^{-2}$ ), $E \sim 50 \mathrm{~V} \mathrm{~cm}^{-1}$ and $v^{\prime} \sim 0.1 \kappa$ this yields $L \sim 10^{4} \mathrm{~cm}^{-2}$-which is similar to the values for current-generated tangles reported previously [10]. Unfortunately, in the experiments reported in this paper, the values of $L$ could not be measured independently; so the verification of (11) should await future tests.

On a microscopic level, each trapped ion under an applied force $e \mathbf{E}$ generates perturbations of the vortex line. There are three different types of perturbations, depending on the particular projection of the force on the directions of the line and its binormal (if it is bent at some radius $R$ like, for example, a vortex ring). The component of the force that is normal to the line and directed along the binormal results 
in gradual self-similar ballooning out $[40,41]$ (or in for the opposite direction of the binormal) of the segment; as a result the ion mainly drifts with the segment's selfinduced velocity. The component of the force that is normal to both vortex line and its binormal results in bending (rotating) the segment while no drift of the ion in the field direction occurs. The component of the force along the vortex line should, in principle, result in accelerated sliding of the ion in the direction of the force. However, the instability with respect to emitting Kelvin waves will inevitably result in an effective tangential drag force that will saturate the ion's drift velocity at some $\sim 10 \mathrm{~ms}^{-1}$ $[28,29]$. We see that in the first case (binormals to vortex segments aligned with the field) the work $e E v_{q}$ of charge drifting with the segment goes into expanding the length of this smooth segment. In the third case (vortex segments aligned with the field) the work on the rapidly sliding ions goes directly into generating smaller-scale Kelvin waves. Thus, the presence of a certain predominant polarization of the tangle might strongly affect the drift velocity of trapped ions, the rate of energy release, the rate of energy dissipation (as the released energy is injected into flow at different length scales) and the self-sustained energy spectrum of QT in the wavenumber space.

\subsection{Momentum Transfer to the Tangle}

Propulsion of helium through the momentum transfer from the injected (non-trapped) ions to the normal component (and then, via mutual friction, to the vortex tangle) was used in the 70-s by Mehrotra et al. [43] and recently in our laboratory [10]. For ions moving at velocity $\mathbf{v}_{q}$ and maintaining the current density $\mathbf{j}$ in field $\mathbf{E}$, the rate of momentum transfer per unit volume is $\mathbf{f}_{q}=q \mathbf{E}=j v_{q}^{-1} \mathbf{E}$. With a well-defined value of ion mobility $\mu$ such that $\mathbf{v}_{q}=\mu \mathbf{E}$, this volume force on the liquid helium becomes $\mathbf{f}_{q}=\mu^{-1} \mathbf{j}$. At the low temperatures and electric fields that we are interested in, all ions are permanently trapped on vortex lines. The force on the charge will still transfer momentum to the tangle and will hence be able to efficiently propel it along. The question that has to be investigated is the velocity $v_{q}(E)$ of trapped ions when they are drifting through (or with) the tangle in which they are trapped. Some observations of an effective mobility, $\mu_{0} \sim 6 \times 10^{-3} \mathrm{~cm}^{2} \mathrm{~V}^{-1} \mathrm{~s}^{-1}$, in charged tangles surrounding a field-emission tip at high voltages were made in 70-s by Phillips and McClintock [22]. Obviously, the efficiency of momentum transfer and forcing of the tangle by the ions depend on the velocities of the ions $v_{q}$ and of the tangle $v_{L}$. We envisage two limiting cases:

1. $v_{q}-v_{L} \ll v_{L}$. In this case the ions almost do not "slip" relative to the tangle. This corresponds to mostly polarized loops with rare reconnections, but with substantial overlap, so that the superfluid velocity is reasonably flat on length scales greater than $\ell=L^{-1 / 2}$. As a result, the momentum transfer becomes efficient, the ions follow the tangle and the drift velocity $v_{L} \approx v_{q}$ increases with increasing force (unlike the case of isolated quantized vortex rings that slow down due to entraining more liquid while gaining more energy). For a very high density of polarized loops the main contribution to energy and momentum will be from the mean-field superfluid velocity, $v_{\mathrm{s}} \approx 0.5 \kappa L \bar{R}$, where $\bar{R}$ is the typical radius of loops. If reconnections are not too frequent, and the charge slip is negligible, the dissipation is relatively small. We thus can have an accelerated motion of the vortex tangle. 
2. An opposite case is when $v_{q} \gg v_{L}$. Here ions rapidly move past a slow tangle. The momentum transfer per ion is greatly reduced while most of the work of the electric field goes not into accelerating the tangle but into the dissipative processes that result from strong forcing of the tangle at small "quantum" length scales $<\ell$. This is the state described in the previous section.

We can speculate what individual events of ion motion through the tangle are like. These can be fast sliding of trapped ions along vortex segments in the direction of the field until they get stuck in a segment normal to the field (this fast sliding should necessarily generate Kelvin waves that, when growing non-linear, might themselves become "traps" of ions in the shape of Hashimoto solitons). After such a quick dash forward, the ions slow down and wait until the next reconnection event in their vicinity creates another vortex segment along the field direction-to slide forward again. As after every reconnection event the ions typically slide forward the distance $\sim \ell$ and then wait until the next reconnection event which are expected to happen at the rate $\sim \kappa \ell^{-2}$, the mean slip velocity should be $v_{\mathrm{s}} \sim \kappa \ell^{-1}$. An alternative mechanism might be the ballooning out of a small charged vortex ring (CVR) detached following a self-reconnection process of a vortex line caused by some strong local non-linear perturbation. Such a ring should predominantly propagate forward in the direction of the field until it grows big enough to get reconnected with the tangle when its radius becomes $\sim \ell$; then, with a substantial probability, the trapped ion creates another small ring - and the process repeats itself. In the meantime the ions propagate the distance $\sim \rho_{\mathrm{s}} \kappa^{2} \ell(e E)^{-1}$ in time $\sim \rho_{\mathrm{s}} \kappa \ell^{2}(e E)^{-1}$; this results in the characteristic velocity $\sim \kappa \ell^{-1}$.

We can see that both speculative mechanisms predict the charge slip in the direction of the electric field $\mathbf{E}$ at a terminal velocity of order $v_{s} \sim \kappa \ell^{-1}$ which is weakly dependent on the magnitude of the field $E$. The important prerequisite for the above considerations is the availability of numerous segments of vortex lines which are non-orthogonal to $\mathbf{E}$.

\section{Experimental Results}

\subsection{Experimental Cell}

The experimental cell was described elsewhere [44]. In short (see Fig. 1), the "drift volume" of liquid helium has a shape of a cube of $d=4.5 \mathrm{~cm}$ side, confined by metal electrode plates. In all experiments presented in this paper, the ions are injected by a field-emission tip through a gridded circular hole in the center of the bottom plate and detected at the collector electrode behind a similar gridded opening in the top plate. The temperature is monitored by a calibrated resistance thermometer bolted to the outer body of the brass container with the cell filled with isotopically-pure liquid ${ }^{4} \mathrm{He}$ [45] at pressure 0.1 bar. The container is rigidly attached to the bottom of the mixing chamber of the dilution refrigerator that can be set in continuous rotation around its vertical axis.

When applying the "driving field" by setting the potential difference $V$ between the injector and collector plates, the side plates are maintained at half the potential, $0.5 \mathrm{~V}$, relative to the injector plate. The electric field inside the drift space is 

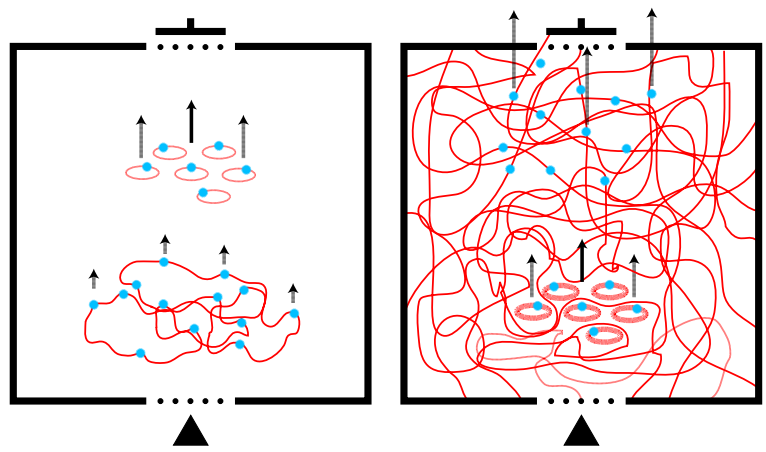

Fig. 1 (Color online) Sketches of the experimental cell, with the injector at the bottom and collector at the top, illustrating two types of experiments. On the left, ions are briefly injected into virgin helium; some of them arrive at the collector with CVRs (after time $t_{2}$ ) while the others arrive as slower moving entangled charged vortex loops (after $t_{3}$ ). On the right, the ions are briefly injected into a dense tangle occupying all drift space; some ions now arrive much faster (after time $t_{1}$ ) while the rest arrive with CVRs and also with charged entangled loops (not shown)

hence moderately non-uniform; its numerically-calculated value on the vertical axis is changing from $1.3 \mathrm{Vd}^{-1}$ at the injector plate to $0.8 \mathrm{Vd}^{-1}$ in the center and back to $1.3 \mathrm{Vd}^{-1}$ at the collector plate. In what follows, we quote the mean electric field defined as $E \equiv V d^{-1}$.

We can separately monitor the currents arriving at the collector, its Frisch grid, the flat metal plate around the collector grid, and the side plates. These measurements give important information on the angular distribution of currents due to ions arriving with vortices but at different times (and hence by different means, say, as isolated charged vortex rings or entangled charged vortex loops).

\subsection{Injection Details}

To be field-emitted from a cold metal [46, 47] into liquid helium, an electron has to tunnel under a barrier of several $\mathrm{eV}$ that is composed of the work function of the metal, excess energy $\sim 1 \mathrm{eV}$ of being surrounded by strongly-repulsive helium atoms, and the attraction to its image charge inside the injector maintained at fixed potential $V_{\text {tip }}$ relative to that of the grid it faces $[48,49]$. For acceptable tunneling rates, the extracting electric field should be of order $E_{\mathrm{ex}} \sim 10^{8} \mathrm{~V} \mathrm{~cm}^{-1}$; this is usually achieved by applying a high voltage $V_{\text {tip }}$ to a sharp metal tip of small radius of curvature $a$ so that $E_{\text {ex }}=V_{\text {tip }} a^{-1}$. In this experiment we use a tungsten tip, electro-etched by a convenient technique [50]. The effective value of $a$ was found to increase with time. For instance, in the initial experiments (Fig. 2) the total current of order $I_{\text {tip }}=$ $10^{-13}$ A could be emitted at voltages of $V_{\text {tip }}=280 \mathrm{~V}$, while after more than three years of experiments and occasional abuse by high currents the voltage $V_{\text {tip }} \approx 400 \mathrm{~V}$ is now necessary to emit $I_{\text {tip }}=10^{-13} \mathrm{~A}$.

Every injected electron, of energy $\sim 300 \mathrm{eV}$, is expected to quickly lose most of its energy to inelastic collisions with helium atoms, producing electron-ion pairs $\mathrm{e}^{-}+\mathrm{He}^{+}$and excimer molecules $\mathrm{He}_{2}^{*}$ along its track. Some positive ions might not 


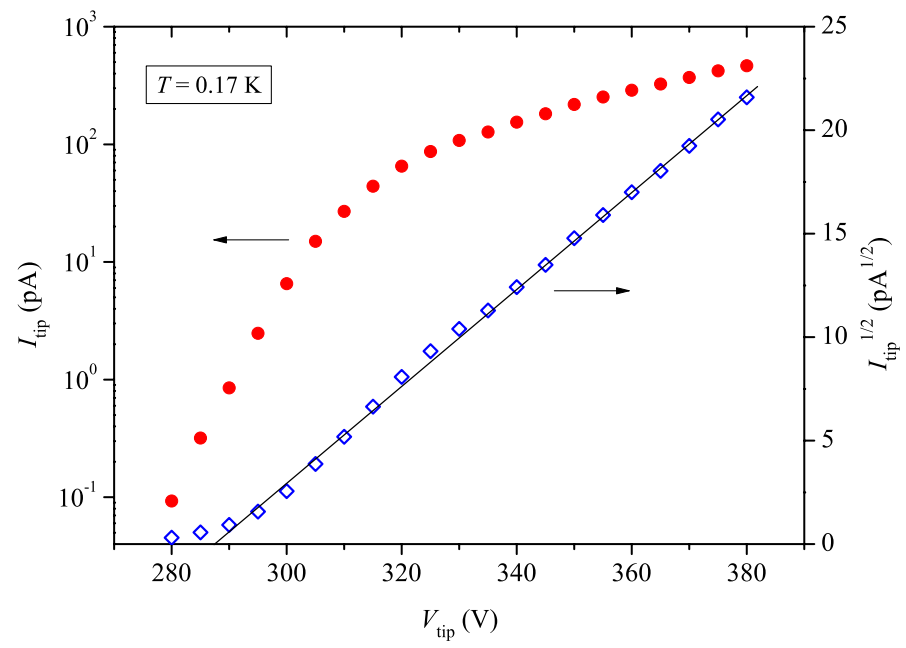

Fig. 2 (Color online) Current collected by the injector grid for different values of steady tip voltage $V_{\text {tip }}$. $T=0.17 \mathrm{~K}, E=0$. The same data $I_{\text {tip }}$ are plotted in logarithmic scale (red solid circles, left axis) and as $I_{\text {tip }}^{1 / 2}$ (blue open diamonds, right axis)

recombine with an electron but get pulled back to the injector tip- thus enabling certain enhancement of the yield of electrons. Each remaining electron slows down via emission of rotons and phonons and eventually, within $\sim 10^{-11} \mathrm{~s}$, self-localizes in a spherical void of radius $\approx 19 \AA[26,51]$. The electron's zero-point kinetic energy in such a void is $\sim 0.2 \mathrm{eV}$.

It is known [52] that, at zero pressure, an electron nucleates a vortex ring at velocity of $34 \mathrm{~m} / \mathrm{s}$ and then gets trapped by the ring's core and moves with that ring [20] - the charged ring grows (and hence slows down) as it is propelled by the electric field between the injector tip and grid. We would expect this to happen within the time $\sim 10^{-9} \mathrm{~s}$ and distance from the tip $\sim 100 \AA$.

Much of the injected current $I_{\text {tip }}$ terminates on its grid while only a fraction, roughly proportional to the field $E \sim V d^{-1}$ on the other side of the grid, continues through the main drift space towards the collector. The current to the injector grid is shown in Fig. 2 for various steady $V_{\text {tip }}$. The current initially increases exponentially with $V_{\text {tip }}$, but this growth slows down for currents above $\sim 10^{-12} \mathrm{~A}$, switching to $\propto\left(V_{\text {tip }}-V_{0}\right)^{2}$ dependence. This observation confirms the reported earlier onset of space-charge-limited regime at high injected currents [22, 49]. Even though our typical injection potentials $V_{\text {tip }}$ were one order of magnitude smaller than those used by Phillips and McClintock [22], the general observations are very similar. As they discussed, the change of the regime might be related to the fact that a tangle of charged vortex lines is created near the injector tip; the charge transport through the tangle being described by some effective mobility. This considerably slows down the removal of the space charge from the tip and the negative feedback of the field due to the space charge controls the emission rate.

At low intensity of injection $I_{\text {tip }}$, each charged ring would travel all the way from the tip to the grid without ever reconnecting with other rings. This would result in 


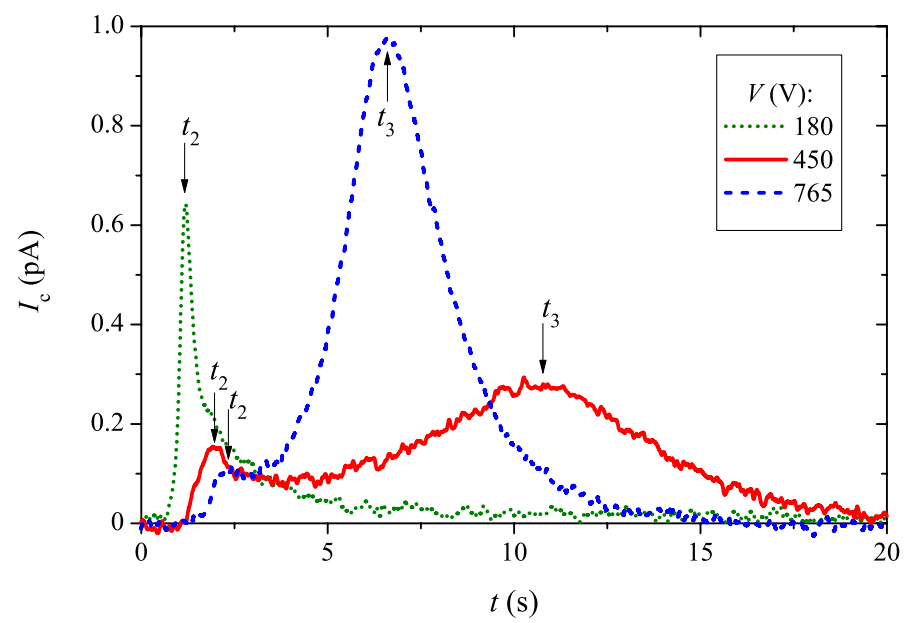

Fig. 3 (Color online) Records of the collector current, following injection pulses of the duration $\Delta t=0.15 \mathrm{~s}$ and intensity $I_{\text {tip }}=7 \times 10^{-12} \mathrm{~A}$, into virgin helium held at temperature $0.19 \mathrm{~K}$ and three different drift voltages $V$. The origin of time coincides with the beginning of the injection pulses

strictly singly-charged rings that gained energy from the field in which they traveled from the point of nucleation. This is in agreement with our observations of mostly singly-charged rings, that enter the drift space with the initial radius $R_{0} \sim 0.9 \mu \mathrm{m}$ (see Sect. 4.1) that corresponds to their initial energy of $\sim 40 \mathrm{eV}$, fairly independent of $I_{\text {tip }}$ and $V$. However, a small fraction of rings appear to be charged by more than one electron (at $T<0.4 \mathrm{~K}$, rings with up to four trapped electrons were observed). This might be related to the rare case of reconnections of charged vortex rings upon mutual collisions before reaching the injector grid.

\subsection{Main Observations}

Two examples of the collector current following a short injection pulse of duration $\Delta t$ are shown in Figs. 3 and 4.

In Fig. 3, ions are injected into nearly virgin helium after vortices from previous experiments have mostly decayed. Three different records correspond to three different driving voltages $V$ but the same current emitted by the tip (yet, the currents injected through the grid into the drift voltage are different as they are roughly proportional to $V$ ). On both records, the first arriving pulse of charge, at times $t_{2}=1-2 \mathrm{~s}$, is carried by isolated charged vortex rings (their arrival time grows with increasing voltage $V$, i.e. rings' energy, as expected), while the second one, at times $t_{3} \sim 10 \mathrm{~s}$, is carried by a tangle of charged vortex loops resulting from occasional reconnections of the initial charged vortex rings (its arrival time decreases with $V$ ).

In Fig. 4, the collector current at short times is shown for short injections into a developed volume-filling vortex tangle maintained by repetitive injection pulses fired every one second. One can see the appearance of a faster peak of collector current, at times $t_{1} \sim 0.3 \mathrm{~s}$ (coexisting with that carried by isolated charged vortex rings arriving later at time $t_{2}$ ) that requires the presence of a continuous vortex tangle between the 


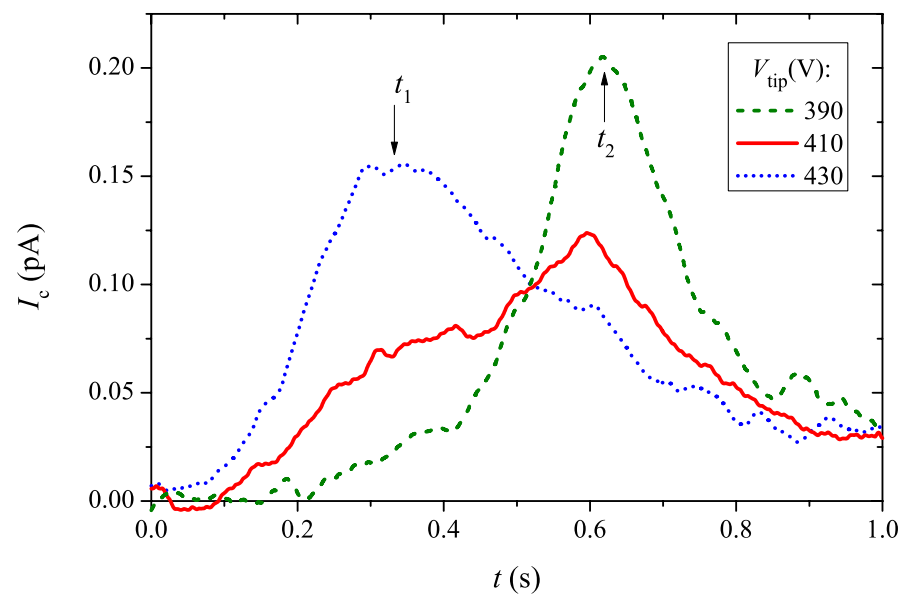

Fig. 4 (Color online) Records of the collector current, through the drift space held at voltage $V=45 \mathrm{~V}$ during repetitive pulses of the injected current of duration $\Delta t=0.2 \mathrm{~s}$ and repetition period of $1 \mathrm{~s}$. The origin of time coincides with the beginning of an injection pulse. The decaying current, mainly due to the "tail" from the previous peak, is subtracted (a small correction). The first peak of the collector current, that only appears at high injection currents, is rather broad: its leading edge arrives after about $0.1 \mathrm{~s}$ while the majority of charge takes $t_{1} \sim 0.3 \mathrm{~s}-0.5 \Delta t=0.2 \mathrm{~s}$ to arrive. The second peak, that is only visible at smaller injection currents, is relatively more compact; its main charge arrives after $t_{2}=0.62 \mathrm{~s}-0.5 \Delta t=0.52 \mathrm{~s}$ with spread at the $50 \%$ level of $\pm 0.09 \mathrm{~s}$

injector and collector. We would interpret this mechanism of charge transport as a rapid charge transfer through a vortex tangle. This mechanism seems to be strongly non-linear as no clear dependence of the transport time on the applied voltage (and injected charge density) was found so far.

We thus observe three distinct mechanisms of transporting ions trapped on vortex lines (Fig. 5) - all much slower than if the ions were "free" and move at the Landau velocity $v_{L}=60 \mathrm{~m} / \mathrm{s}$ reaching the collector in just $0.8 \mathrm{~ms}$. The first (fastest) mechanism (with a range of drift times $t_{1}=0.1-0.4 \mathrm{~s}$, fairly insensitive to $V$ ) only exists when there is already a continuous vortex tangle filling the space between the injector and collector; we tentatively associate it with trapped ions moving through a slowly moving tangle. The second pulse (at $t_{2}=1-3 \mathrm{~s}, t_{2}$ increasing with $V$ ) agrees with the arrival of isolated CVRs. The third one (at $t_{3}=6-20 \mathrm{~s}, t_{3}$ decreases with $V$ ) corresponds to trapped charge slowly drifting either through the tangle or together with the tangle of vortices.

As we can independently measure currents to the collector, its grid, the surrounding electrode (plate) and side electrodes, we can quantify the radial spread of the charge upon crossing the drift space. In Fig. 6 we plot the ratio of the integral charge that arrived within the radius $10 \mathrm{~mm}$ off the central axis (i.e. collected by the collector, $Q_{\mathrm{c}}$, and its grid with grid's frame, $Q_{\mathrm{g}}$ ) to the charge that arrived outside this radius ( $Q_{\mathrm{p}}$, collected mainly by the outer plate hosting the collector in its center), $Q_{\mathrm{p}}\left(Q_{\mathrm{c}}+Q_{\mathrm{g}}\right)^{-1}$. Clearly, the CVRs in small electric fields spread within a considerable radius of about twice the $10 \mathrm{~mm}$, while a huge majority of charge arriving with the charged tangle is collected well-within the collector grid's frame of radius 


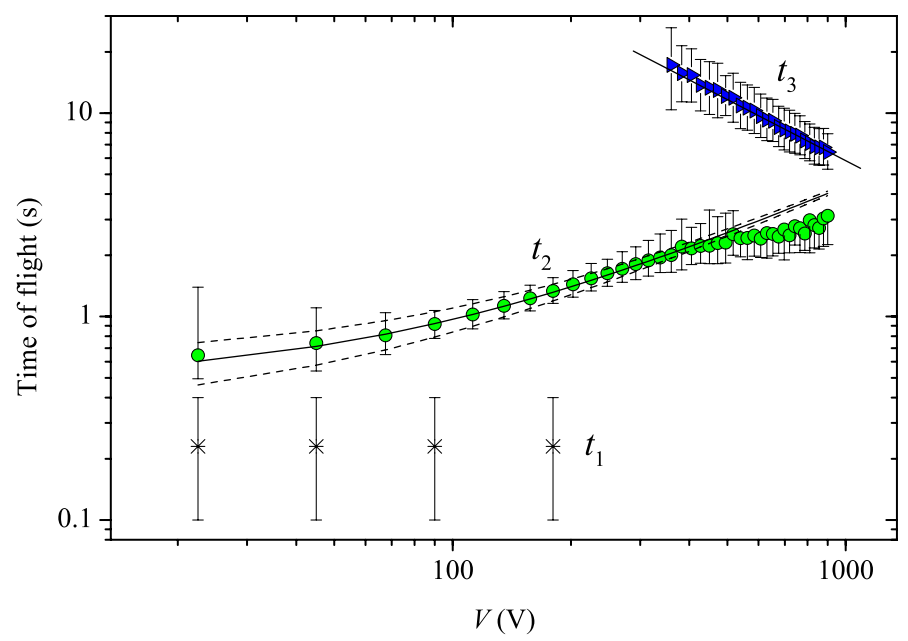

Fig. 5 (Color online) The times of flight, $t_{1}(*), t_{2}$ (green $\left.\circ\right)$ and $t_{3}($ blue $\triangleright)$, corresponding to the positions of maxima of three distinct peaks of the collector current relative to the middle of the injection pulse, $0.5 \Delta t$, - after an injection pulse of duration $\Delta t=0.15 \mathrm{~s}$ into the drift region maintained at voltage $V$ and temperature $T=0.17 \mathrm{~K}$. To observe $t_{2}$ and $t_{3}$, the ions were injected into virgin helium, while for $t_{1}$ they were injected into a space-filling tangle generated by a prolonged intensive ion injection that was stopped 0.8 seconds before firing the probe pulse. The bars at symbols indicate the width of the particular peak of current at the $50 \%$ level (at high $V$, when the peak due to CVRs at $t_{2}$ becomes small and gets too close to the much bigger peak due to the charged tangle at $t_{3}$, unambiguous determination of its trailing edge becomes impossible and hence is not shown). The calculated theoretical times of flight $t_{2}$ for isolated quantized CVRs in the given electric field are shown for different values of the initial radius of rings: upper dashed line $R_{0}=1.2 \mu \mathrm{m}$, solid line $R_{0}=0.85 \mu \mathrm{m}$, lower dashed line $R_{0}=0.6 \mu \mathrm{m}$. Another solid line through the data points for the time of flight of charges tangle indicate the dependence $t_{3} \propto V^{-1}$

$10 \mathrm{~mm}$. This reflects the fact that CVRs are injected into the drift space at a large solid angle and with certain impulse $\pi \rho \kappa R_{0}^{2}$; large electric field can help confining them more near the axis but within limits. In a very different fashion, the charged tangle propagates very much along the central axis, especially in large fields. This is helped by the fact that in large fields most injected CVRs get entangled very near the injector, i.e. before spreading much from the axis; after that the memory of the individual impulses of CVRs is lost and the charge propagates strictly in the direction of the propelling electric field.

\section{Discussion}

\subsection{Isolated Charged Vortex Rings}

An isolated vortex ring has its energy nearly proportional to the radius while the velocity of self-induced motion inversely proportional to radius [15, 20]. The presence of one a trapped ion in an electric field normal to the ring's plane, causes it to gain energy while maintaining nearly circular shape in a self-similar way [40, 41]. Hence, thanks to the precise conservation of quantized circulation, $\kappa=h m_{4}^{-1}$, the energyvelocity relation becomes of type $\mathcal{E} \propto v^{-1}$ [20]. The measured time of flight $t_{2}$ (the 


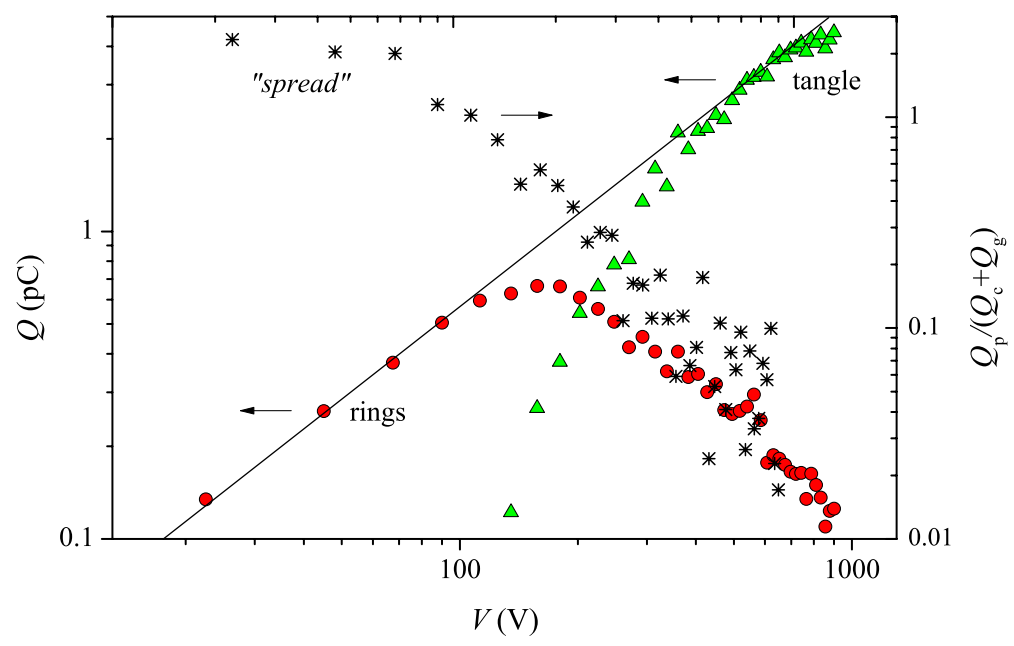

Fig. 6 (Color online) Comparisons of the amounts of charge, traveling by different means and into different destinations, after a single injection pulse of duration $\Delta t=0.2 \mathrm{~s}$ and intensity, $I_{\text {tip }}=2 \times 10^{-10}$ A into virgin helium of temperature $T=0.2 \mathrm{~K}$ in different applied voltages $V$. Left axis: red circles (rings)-the integrated charge arriving at collector with the CVR peak $\left(t_{2}\right)$; green triangles (tangle) - integrated charge arriving with slower charged vortex tangle $\left(t_{3}\right)$; the solid line for the total collector charge $Q \propto V$ guides the eye through the cross-over region at $E \sim 50 \mathrm{~V} / \mathrm{cm}$ between the CVR-dominated and tangle-dominated charge transport. Right axis: asterisks ("spread") $-Q_{\mathrm{p}}\left(Q_{\mathrm{c}}+Q_{\mathrm{g}}\right)^{-1}$, where $Q_{\mathrm{p}}, Q_{\mathrm{c}}$ and $Q_{\mathrm{g}}$ are the total charges collected by the outer plate, collector and its grid with the supporting frame, respectively

time interval between the middle of the injecting pulse and the arrival of the maximum of the collector current) as function of drift voltage $V$ agrees well with the expected dynamics of quantized vortex rings of circulation $\kappa$ (Fig. 5) with the mean initial radius of some $R_{0} \sim 0.9 \mu \mathrm{m}$. The width of the arriving pulse is normally comparable to that of the injection pulse $\Delta t$ (usually between 0.15 and $0.5 \mathrm{~s}$ ). At small voltages $V$ the arriving pulses are weak and appear broader (perhaps in part due to a wider range of initial radii $R_{0}$ ). With increasing voltage $V$, the ring's radius and hence velocity become less sensitive to the spread of initial $R_{0}$ and the arriving pulse narrows. At higher injection intensities and durations, some additional narrowing was observed that is probably related to the soliton-like behaviour caused by the negative feedback of the electric field due to the rings on their local velocity (Coulomb repulsion of charged rings acts to narrow their cloud because of the inverse dependence of the ring velocity on its energy) [42]; this was not studied systematically yet. The seed rings, as they appear in the drift space after crossing the injector grid, have a large solid angle of initial velocities (Fig. 6). With increasing driving voltage $V$, more charged rings are being drawn into the drift space, and a larger fraction of rings terminates within the collector grid (Fig. 6).

\subsection{High Density of Rings}

A convenient non-dimensional parameter characterizing the density of vortex rings of nearly the same radii $R$ is $n R^{3}$, where $n$ is their number density. This product depends 
on both the current density $j=e n v(R)$ (for singly-charged rings) and strength of electric field - that directly affects their radius

$$
R(x)=R_{0}+\frac{2 e}{\rho_{\mathrm{s}} \kappa \Lambda} \int_{0}^{x} E\left(x^{\prime}\right) \mathrm{d} x^{\prime},
$$

where $x$ is the distance from the injector grid along the axis towards the collector. As $\int_{0}^{d} E\left(x^{\prime}\right) \mathrm{d} x^{\prime}=V$, the radius the ring attains approaching the collector is

$$
R(d)=R_{0}+\frac{2 e}{\rho_{\mathrm{S}} \kappa \Lambda} V .
$$

With increasing density $n R^{3}$ of injected rings (either by increasing the voltage $V$ or injection current $I_{\text {tip }}$ or both) towards a value of order $10^{-4}$, the pulses of the collector current become broadened and the total charge arriving with these peaks stops growing with $V$ (for the datasets shown in Figs. 5 and 6 this happens at voltage $V>200 \mathrm{~V}$ ); the remaining injected charge arrives later as a long decaying tail of current. At even stronger voltages, the charge associated with CVRs begins to decrease with $V$ (even though the total injected charge keeps growing as $Q \propto V$ ) while the rest of the injected charge arrives as another well-defined peak (labeled " $t_{3}$ " in Fig. 5 and "tangle" in Fig. 6). We interpret this change as the result of occasional strong interactions (either purely hydrodynamic ones at short distances $\sim R$ or reconnections of vortex loops) between the CVRs when their density $n R^{3}$ is high. The broadening of the CVR peak implies that some charged rings now arrive earlier and some later than the isolated monoenergetic rings. The decrease in their numbers and the appearance of the second slower peak reveal that slower moving tangle of charged vortex loops [53] or very slowly moving deformed charged vortex rings [54] are formed as the result of those interactions.

\subsection{Intensive Injection into Strong Field}

Intensive injections into sufficiently strong fields, such that the rings' radii grow quickly, result in the majority of trapped ions left on slowly moving reconnecting loops created not too far from the injector. The time of flight of these ions, $t_{3}$, decreases with increasing of both the applied voltage $V$ (as $t_{3} \propto V^{-1}$, see Fig. 5) and total charge $Q$ that travels on these loops ( $Q \propto V$, see Fig. 6). As function of the total force, $F=Q E \propto V^{2}$, we thus obtain $t_{3} \propto F^{-1 / 2}$.

The "hopping" mechanism of charge transfer through the tangle yields $v_{q}-v_{L} \sim$ $\kappa L^{1 / 2}$. After plugging in the result of (11), we arrive at

$$
v_{q}-v_{L} \sim\left(\frac{j E}{\rho_{\mathrm{s}} \kappa^{3}\left(v^{\prime} / \kappa\right)}\right)^{1 / 2} .
$$

For $v^{\prime}=0.1 \kappa$ and our typical experimental values of $j \sim 10^{-12} \mathrm{~A} \mathrm{~cm}^{-2}$ and $E \sim$ $100 \mathrm{~V} \mathrm{~cm}^{-1}$, this yields $\sim 0.5 \mathrm{~cm} \mathrm{~s}^{-1}$, and hence $t_{3} \sim 9 \mathrm{~s}-$ which is close to the experimental values. Moreover, as the charge $Q$ injected into the cell is proportional to the current density, $Q \sim j \sigma^{2} \Delta t$ (assuming constant radial spread of the charge 
$\sigma \sim 1 \mathrm{~cm}$ ), this steady-state velocity would result in the time of flight of (assuming $\left.v_{L} \ll v_{q}\right)$

$$
t_{3} \sim\left(\frac{d Q V}{\rho_{\mathrm{s}} \kappa^{3}\left(\nu^{\prime} / \kappa\right) \sigma \Delta t}\right)^{1 / 2},
$$

in agreement with the experiment.

Thus the observed dependence $t_{3} \propto F^{-1 / 2}$ might correspond to a terminal drift velocity of a steady state set by a cascade of energy flux per unit volume $f_{q} v_{q}$ equal to the rate of energy dissipation $v^{\prime}(\kappa L)^{2}$ (by the way, for classical turbulence at high Reynolds number, the drag caused by the turbulent wake past a moving body is also expected to scale as $F \propto v^{2}$ ). However, the interpretation in the spirit of the simplistic model a slow steady-state tangle sustained by the ion current in electric field has several problems. It requires the presence of some continuous vortex tangle ahead of the propagating ions, while we believe that in the experiments in high electric fields most ions find themselves stuck on slow vortex loops not too far from the injectorwithout much vortex tangle ahead of them. If this is the case, in order to reach the collector the trapped ions should force the vortex loops to propagate forward. The fact that the peak of the collector current at $t_{3}$ is rather broad tells that the trapped ions most probably passively ride on the drifting loops without much slipping through the tangle - otherwise most of them would just quickly slide forward through the existing tangle into its front.

In fact, there might be an alternative interpretation. We observed that the flow is actually not steady but keeps evolving (perhaps, accelerating and expanding) during much longer times ( $\sim 300 \mathrm{~s}$ or more). This implies that the exponent $\approx 1 / 2 \mathrm{might}$ also arise from a uniformly accelerated mass of fluid under a constant force. These phenomena, along with a modification of the model of Sect. 2.2 for non-stationary tangles and substantial large-scale flow, will be discussed in forthcoming publications.

\section{4 "Conductive" Tangles After Continuous Injections}

Finally, it was observed that, upon an extended injection of ions at relatively high current and driving voltage, some fraction of ions begins to travel through the drift space much faster than even isolated rings. The characteristic time of travel, $t_{1} \sim 0.2 \mathrm{~s}$, corresponds to the mean drift velocity of $\sim 20 \mathrm{~cm} \mathrm{~s}^{-1}$. While this is much slower than the limiting velocity of ions traveling along straight vortex lines, $\sim 10 \mathrm{~m} \mathrm{~s}^{-1}$ $[28,29]$, it is much faster than the expected drift velocity for the "reconnectionassisted hopping" mechanism discussed in Sect. 2.3, $\sim \kappa L^{1 / 2}=0.1 \mathrm{~cm} / \mathrm{s}$, if $L=$ $10^{4} \mathrm{~cm}^{-2}$.

So far, a necessary prerequisite for the appearance of the fast first peak, was a prolonged injection (either continuous or quasi-continuous, in the form of many pulses with the repetition frequency $\sim 1 \mathrm{~Hz}$ ) of ions into the drift region under an applied electric field. The duration of the injection, necessary for the fast peak to appear and settle at its shortest time of flight, increases with decreasing the injected current (although no systematic investigation of this has been conducted so far). An application of fast rotation around an axis normal to the flow of charge was found to suppress this 
fast train of current while simultaneously restoring the peak due to isolated charged vortex rings. On the other hand rotation around the direction of ion motion was found to enhance this fast train [55]. These observations seem to hint that this mechanism relies on the presence of a dense tangle of vortex lines throughout all space between the injector and collector-with a substantial fraction of vortex segments having a non-zero projection onto the direction of drift. Finally, the relevance of a polarized bundle of vortex lines for the fast transport can be corroborated by the earlier observation [35] that a strongly polarized tangle of vortex lines, generated not by the current injection but by the spin-down of the container to rest, can also support a fast arrival of ions.

\section{Summary and Outlook}

Injected ions, trapped on vortex lines in superfluid ${ }^{4} \mathrm{He}$ at $T<1 \mathrm{~K}$, provide a versatile tool for generating different states of QT and sampling their dynamics. Time-of-flight measurements revealed at least two distinct states of vortex tangles: one associated with slow transport of trapped ions and another allowing a much faster transport of ions through the tangle. We tentatively interpret the former as a tangle of strongly polarized loops that mainly advect the trapped ions with small dissipation, and the latter as a more isotropic tangle, through which ions can propagate rapidly, in a highly dissipative state that can be only maintained at substantial levels of forcing.

Acknowledgements Support was provided by EPSRC under GR/R94855, EP/E001009 and $\mathrm{EP} / \mathrm{H} 014691$.

\section{References}

1. R.P. Feynman, in Progress in Low Temperature Physics, ed. by C.J. Gorter, vol. I (North-Holland, Amsterdam, 1955), p. 17

2. W.F. Vinen, J.J. Niemela, J. Low Temp. Phys. 128, 167 (2002)

3. W.F. Vinen, J. Low Temp. Phys. (this volume)

4. W.F. Vinen, Proc. R. Soc. Lond. A 240, 114 (1957)

5. W.F. Vinen, Proc. R. Soc. Lond. A 240, 128 (1957)

6. W.F. Vinen, Proc. R. Soc. Lond. A 242, 493 (1957)

7. W.F. Vinen, Proc. R. Soc. Lond. A 243, 400 (1958)

8. W.F. Vinen, Vortex lines in liquid helium II, in Prog. Low Temp. Phys., vol. III, ed. by C.J. Gorter (Elsevier, Amsterdam, 1961), pp. 1-57

9. G.E. Volovik, JETP Lett. 78, 533 (2003)

10. P.M. Walmsley, A.I. Golov, Phys. Rev. Lett. 100, 245301 (2008)

11. E.V. Kozik, B.V. Svistunov, Phys. Rev. B 77, 060502 (2008)

12. V.B. Eltsov, R. de Graaf, R. Hanninen, M. Krusius, R.E. Solntsev, V.S. L'vov, A.I. Golov, P.M. Walmsley, in Progress in Low Temperature Physics ("Quantum Turbulence”), vol. XVI, ed. by M. Tsubota (North-Holland, Amsterdam, 2008), pp. 45-146

13. A.I. Golov, P.M. Walmsley, J. Low Temp. Phys. 156, 51 (2009)

14. K.W. Schwarz, Phys. Rev. B 38, 2398 (1988)

15. R.J. Donnelly, Quantized Vortices in Helium II (Cambridge University Press, Cambridge, 1991)

16. V.S. L'vov, S.V. Nazarenko, O. Rudenko, J. Low Temp. Phys. 153, 140 (2008)

17. H.E. Hall, W.F. Vinen, Proc. R. Soc. Lond. Ser. A 238, 204 (1956)

18. G. Careri, F. Scaramuzzi, J.O. Thomson, Nuovo Cimento 18, 957 (1960)

19. G. Careri, W.D. McCormic, F. Scaramuzzi, Phys. Lett. 1, 61 (1962) 
20. G.W. Rayfield, F. Reif, Phys. Rev. 136, A1194 (1964)

21. E.J. Yarmchuk, M.J.V. Gordon, R.E. Packard, Phys. Rev. Lett. 43, 214 (1979)

22. A. Phillips, P.V.E. McClintock, Philos. Trans. R. Soc. Lond. A 278, 271 (1975)

23. S.I. Davis, P.C. Hendry, P.V.E. McClintock, Physica B 280, 43 (2000)

24. K.W. Schwarz, Mobilities of charge carriers in superfluid helium, in Advances in Chemical Physics, vol. 33, ed. by I. Prigogine, S.A. Rice (Wiley, New York, 1975), p. 3

25. C.C. Grimes, G. Adams, Phys. Rev. B 45, 2305 (1992)

26. M. Pi, R. Mayol, A. Hernando, M. Barranco, F. Ancilotto, J. Chem. Phys. 126, 244502 (2007)

27. R.M. Ostermeier, W.I. Glaberson, J. Low Temp. Phys. 20, 159 (1975)

28. W.I. Glaberson, R.M. Ostermeier, Phys. Rev. Lett. 35, 241 (1975)

29. R.M. Ostermeier, W.I. Glaberson, J. Low Temp. Phys. 25, 317 (1976)

30. C.M. Muirhead, W.F. Vinen, R.J. Donnelly, Philos. Trans. R. Soc. Lond. A 311, 433 (1989)

31. D. Kivotides, C.F. Barenghi, Y.A. Sergeev, Phys. Rev. B 77, 014257 (2008)

32. D. Kivotides, C.F. Barenghi, Y.A. Sergeev, Phys. Rev. B 75, 212502 (2007)

33. M.S. Paoletti, M.E. Fisher, K.R. Sreenivasan, D.P. Lathrop, Phys. Rev. Lett. 101, 154501 (2008)

34. P.M. Walmsley, A.I. Golov, H.E. Hall, A.A. Levchenko, W.F. Vinen, Phys. Rev. Lett. 99, 265302 (2007)

35. P.M. Walmsley, A.I. Golov, H.E. Hall, W.F. Vinen, A.A. Levchenko, J. Low Temp. Phys. 153, 127 (2008)

36. K.W. Schwarz, R.J. Donnelly, Phys. Rev. Lett. 17, 1088 (1966)

37. K.W. Schwarz, Phys. Rev. 165, 323 (1968)

38. J.T. Tough, in Progress in Low Temperature Physics, vol. VIII, ed. by D.F. Brewer (North-Holland, Amsterdam, 1982), p. 133

39. H. Adachi, S. Fujiyama, M. Tsubota, Phys. Rev. B 81, 104511 (2010)

40. C. Samuels, R.J. Donnelly, Phys. Rev. Lett. 67, 2505 (1991)

41. M. Tsubota, H. Adachi, J. Low Temp. Phys. 158, 364 (2010)

42. G. Gamota, Phys. Rev. Lett. 31, 517 (1973)

43. R. Mehrotra, E.K. Mann, A.J. Dahm, J. Low Temp. Phys. 36, 47 (1979)

44. P.M. Walmsley, A.A. Levchenko, S.E. May, A.I. Golov, J. Low Temp. Phys. 146, 511 (2007)

45. P.C. Hendry, P.V.E. McClintock, Cryogenics 25, 526 (1985)

46. R.H. Fowler, L. Nordheim, Proc. R. Soc. Lond. 119, 173 (1928)

47. T.E. Stern, B.S. Gossling, R.H. Fowler, Proc. R. Soc. Lond. 124, 699 (1929)

48. D.G. Onn, M. Silver, Phys. Rev. 183, 295 (1969)

49. B. Halpern, R. Gomer, J. Chem. Phys. 51, 1031 (1969)

50. A. Golov, H. Ishimoto, J. Low Temp. Phys. 113, 957 (1998)

51. M. Rosenblit, J. Jortner, J. Phys. Chem. A 101, 751 (1997)

52. G.W. Rayfield, Phys. Rev. 168, 222 (1968)

53. S. Fujiyama, A. Mitani, M. Tsubota, D.I. Bradley, S.N. Fisher, A.M. Guénault, R.P. Haley, G.R. Pickett, V. Tsepelin, Phys. Rev. B 81, 180512 (2010)

54. C.F. Barenghi, R. Hänninen, M. Tsubota, Phys. Rev. E 74, 046303 (2006)

55. P.M. Walmsley, A.I. Golov, A.A. Levchenko, B. White, J. Low Temp. Phys. 148, 317 (2007) 